\title{
Fiducial-Less Respiration Tracking in Radiosurgery
}

\author{
Achim Schweikard ${ }^{1}$, Hiroya Shiomi ${ }^{2}$, Jens Fisseler ${ }^{1}$, Manfred Dötter ${ }^{3}$, \\ Kajetan Berlinger ${ }^{3}$, Hans-Björn Gehl ${ }^{4}$, and John Adler ${ }^{5}$ \\ 1 Informatik, Universität Lübeck, 23538 Lübeck, Germany \\ 2 Div. of Multidisciplinary Radiotherapy, Osaka University Graduate School of \\ Medicine, Japan \\ 3 Informatik, Technische Universität München, 85748 München, Germany \\ 4 Radiology, Universität Lübeck, 23538 Lübeck, Germany \\ ${ }^{5}$ Neurosurgery, Stanford University Medical Center, Stanford, CA. 94304, USA
}

\begin{abstract}
Respiratory motion is difficult to compensate for with conventional radiotherapy systems. An accurate tracking method for following the motion of the tumor is of considerable clinical relevance. We investigate methods to compensate for respiratory motion using robotic radiosurgery. Infrared emitters are used to record the motion of the patient's skin surface. The position of internal gold fiducials is computed repeatedly during treatment, via x-ray image processing. We correlate the motion between external and internal markers. From this correlation model we infer the placement of the internal target. 15 patients with lung tumors have recently been treated with a fully integrated system implementing this new method. In this work we extend our method to tracking without implanted fiducials. We propose to use deformation algorithms on CT data sets combined with registration of digitally reconstructed radiographs to obtain intermittent information on the target location. This information is then combined with our basic correlation method to achieve real-time tracking. The term $7 \mathrm{D}$ registration is coined to describe the underlying method for performing this task.
\end{abstract}

\section{Introduction}

Radiosurgery uses a moving beam of photon radiation to destroy tumors. High precision radiosurgery has been limited to brain tumors, since stereotactic fixation is difficult to apply to tumors in the chest or abdomen. To apply radiosurgical methods to tumors in the chest and abdomen, it is necessary to take into account respiratory motion. Respiratory motion can move the tumor by more than $1 \mathrm{~cm}$. Without compensation for respiratory motion, it is necessary to enlarge the target volume with a "safety margin". For small targets, an appropriate safety margin produces a very large increase in treated volume. 


\section{Related Work}

Intra-treatment displacements of a target due to respiration have been reported to exceed $3 \mathrm{~cm}$ in the abdomen, and $1 \mathrm{~cm}$ for the prostate [4], [8]. Conventional radiation therapy with medical linear accelerators (LINAC-systems) uses a gantry with two axes of rotation movable under computer control [11. This mechanical construction was designed to deliver radiation from several different angles during a single treatment. It was not designed to track respiratory motion. Respiratory gating is a technique for addressing this problem with conventional LINAC radiation therapy. Gating techniques do not directly compensate for breathing motion. I.e., the therapeutic beam is not moved during activation. Instead the beam is switched off whenever the target is outside a predefined window.

Tada and Minakuchi et. al. 9] report using an external laser range sensor in connection with a LINAC-based system for respiratory gating. This device is used to switch the beam off whenever the sensor reports that the respiratory cycle is close to maximal inhalation or maximal exhalation.

In an earlier paper [7, we investigated a method for tracking a tumor during treatment with a robotic arm. Stereo x-ray imaging is combined with infrared tracking. x-ray imaging is used as an internal sensor, while infrared tracking provides simultaneous information on the motion of the patient surface. While x-ray imaging gives accurate information on the internal target location, it is not possible to obtain real-time motion information from x-ray imaging alone. In contrast, the motion of the patient surface can be tracked with commercially available high speed infrared position sensors. The main idea of our approach is to use a series of images from both sensors (infrared and x-ray) where signal acquisition is synchronized. From such a series of sensor readings and corresponding time-stamps, we can determine a motion pattern. This pattern correlates external motion to internal motion.

Below we describe an integrated system using the new correlation method from [7] (internal versus external fiducials), and outline a concept for extending this work to tracking without implanted fiducials. A major obstacle for reaching the goal of tracking without implanted fiducial markers is that image registration must now be based solely on anatomic landmarks. The standard techniques available are currently not suitable for real-time tracking. We thus present a method for splitting the task of registration into a pre-operative computation phase and an intra-operative phase. As a result, our method does not require elastic registration.

\section{System}

Our system has the following components (Fig. 1). A robot arm (modified Cyberknife system) moves the therapeutic beam generator (medical linear accelerator). The component added to the standard Cyberknife system is an infrared tracking system (BIG Inc., Boulder CO, USA). Infrared emitters are attached to the chest and the abdominal surface of the patient. The infrared tracking system 
records the motion of these emitters. A stereo x-ray camera system (two x-ray cameras with nearly orthogonal visual axes) records the position of internal gold markers, injected through an 18 gauge biopsy needle into the vicinity of the target area under CT monitoring.

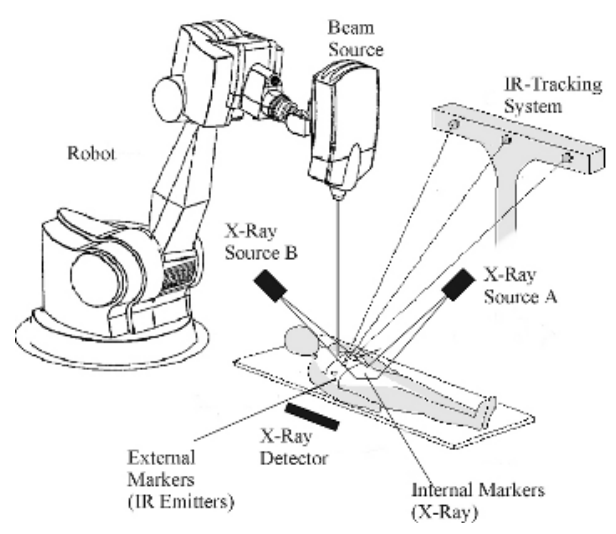

Fig. 1. System overview. A robotic arm moves the beam source to actively compensate for respiratory motion. Infrared surface tracking is correlated to internal x-ray imaging.

The correlation method for computing the position of the internal target has been described in more detail in [7] and is briefly summarized here. Prior to treatment, small gold markers are placed in the vicinity of the target organ. Stereo x-ray imaging is used during treatment to determine the precise spatial location of these gold markers once every 10 seconds.

External markers (placed on the patient's skin) can be tracked automatically with optical methods at very high speed. Updated positions can be transmitted to the control computer more than 20 times per second. However, external markers alone cannot adequately reflect internal displacements caused by breathing motion. Large external motion may occur together with very small internal motion, and vice versa. Similarly, the target may move much slower than the skin surface. Since neither internal nor external markers alone are sufficient for accurate tracking, x-ray imaging is synchronized with optical tracking of external markers. The external markers are small infrared emitters attached to the patient's skin. The first step during treatment is to compute the exact relationship between internal and external motion, using a series of x-ray snapshots showing external and internal markers simultaneously. Each snapshot has a time-stamp which is used to compute the correlation model. 


\section{Tracking Without Fiducials}

The method described above requires x-ray opaque fiducials to be placed in the vicinity of the target. We extend our method in such a way that impanting fiducials is no longer needed. This extension works as follows:

Prior to treatment two CT scans are taken. One scan shows inhalation, one shows exhalation. From this data a series of intermediate CT scans is computed. This yields a series of $3 \mathrm{D}$ scans, showing the respiration process as a threedimensional motion picture. For each point in this time series, we compute a set of digitally reconstructed radiographs (DRR), (see e.g. [6]). A single DRR is computed by projecting a CT scan from a preselected angle using the known geometry of the x-ray imaging system. Assume we have 12 time steps in our CT 3D motion picture. For each of the 12 time steps, we compute a number of DRRs for a preselected angle.
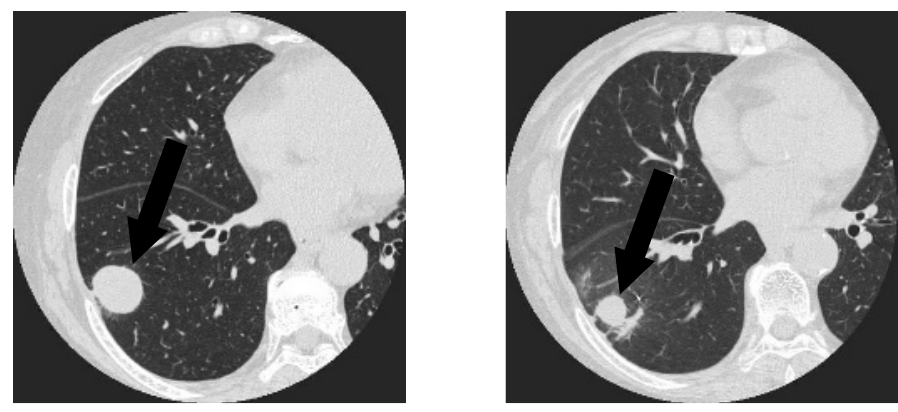

Fig. 2. Treatment of an adeno carcinoma with fiducial based respiration tracking. (Osaka university hospital. Treatment with 39 Gy / 3 Fractions).

During treatment, stereo x-ray images are taken with the stereo x-ray cameras. These images will be called live images. When a new live shot is taken, it is compared to each of the previously computed DRRs. This comparison selects the best matching DRR in the series. Assume this best matching DRR has index number $n$. Each DRR was computed from one CT scan. Then we can identify the CT scan from which this specific DRR (index number $n$ ) was generated. This allows for identifing a time step in the 3D CT series, i. e. a respiration state within the respiratory cycle. Furthermore the given DRR (index number $n$ ) represents a specific angle, under which the present CT scan is seen. This allows for determining the angle under which the patient was seen in the given live shot. Furthermore, computerized comparisons between the live shot and DRRs permit translational shifts to be determined. Overall, if the target is marked in each CT scan, we can identify the exact target location (position, orientation, respiration state) at the time the live shot was taken.

Notice that this gives only intermittent information on the target location. At the time the comparison of all DRRs with the current live shot is completed, 
the target may already have moved. However, our method is nonetheless capable of determining the real-time target position. As in the above fiducial-based correlation method, a real-time sensor is used to report information on the current state of respiration in real time. This information is correlated to the target location computed by the comparison between the live shot and DRRs.

\section{Experiments and Clinical Trials}

Clinical trials of the method in section 3 have been carried out at several institutions, including Cleveland Clinic Foundation and Osaka University Hospital. 15 patients have since been treated at Osaka University Hospital with the fiducialbased method described in section 3 .

\subsection{Clinical Trials}

Figure 3 shows representative results for one clinical case. The figure shows the total correlation error. Thus, based on the correlation model (section [3), we compute the current position of the target based on the external infrared sensor signal alone. At this same time point, we also acquire a pair of x-ray images. We then plot the distance in $\mathrm{mm}$ from the placement inferred by the correlation model and the actual placement determined from the implanted fiducial markers in the image. The top curve in this figure shows the corresponding target excursion.

\subsection{Generating Synthetic Intermediate CT Scans by Deformation}

In vitro trials have been carried out to determine practicality of the fiducial-less method described above. In a first experiment, deformation methods for computing intermediate tomographic scans were tested. The goal of the experiment was to assess the practicality of the deformation sub-steps in terms of computing time and accuracy. A variety of methods for elastic registration and deformation (intensity or landmark-based) have been described in the literature (see e.g. [5] for a survey). Given two sets of tomographic data (CT or MR), one in inhale one in exhale position, plus a third "test set" of tomographic data (intermediate point in the respiratory cycle), we wish to verify that the test set indeed matches one of the synthetic 3D data sets computed by deformation. To this end liver liver data sets for several volunteers were acquired. For each of these volunteers, one inhale, one exhale, and up to three intermediate scans were aquired. The images were taken by a T1 sequence in a Siemens Symphony MRT scanner with 1.5 Tesla and gradients of $30 \mathrm{mTesla} / \mathrm{m}$. In each case complete 3D image data sets (40 slices $0.65 \mathrm{~mm}$ of pixel distance within each layer, $2.5 \mathrm{~mm}$ thickness per layer and $2 \mathrm{~mm}$ slice distance) were taken. Aquisition time per complete data set is in the range of $15-25$ seconds.

Three algorithms for deformation with various types of user interaction were implemented: Thirion's demons algorithm [10], thin plate spline warping based 


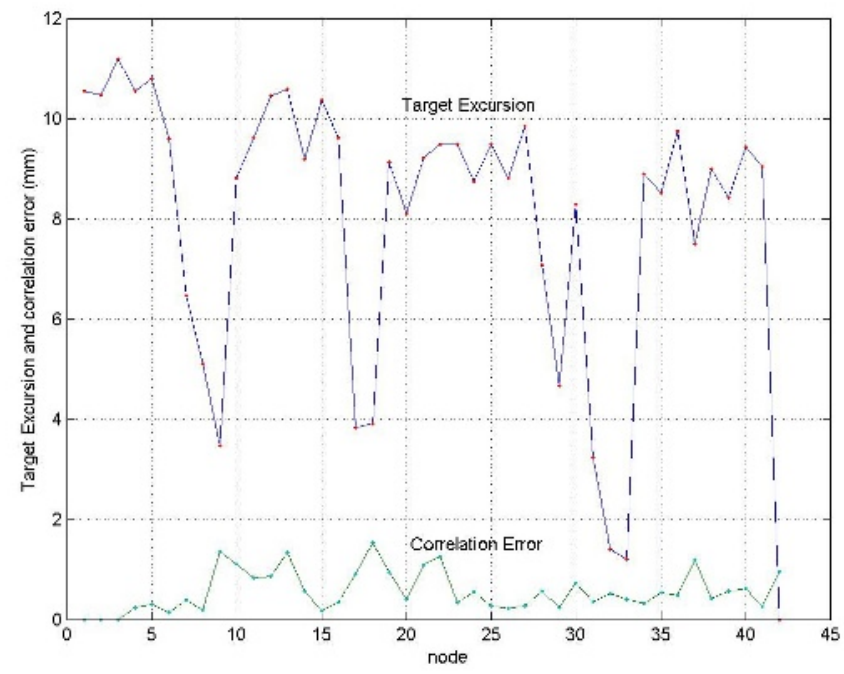

Fig. 3. Total target excursion (top curve), and correlation error (bottom curve) in mm for a clinical case. $\mathrm{x}$-axis: treatment beam direction number (x-ray live shot number), $\mathrm{y}$-axis: error in mm.

on control points placed at landmarks ([1]) and linear interpolation based on control points at landmarks ([2]). The liver volumes were segmented semi-automatically with a modified intelligent scissors algorithm to reconstruct the surface of the liver. This allows for measuring distances between deformed surfaces. Furthermore, given the segmentation, we compute the center of gravity of the segmented liver volume in each case. The total paths travelled by the center of gravity ranged from $21.8 \mathrm{~mm}$ to $46.7 \mathrm{~mm}$ for data stemming from distinct volunteers. From each algorithm we obtain a deformation field allowing to compute a continuous deformation between inhalation and exhalation. We consider the maximum surface distance between the intermediate data set obtained by actual imaging and the data set obtained synthetically by deformation.

Figure 4 shows the maximum surface distance between the synthetic data and the intermediate data set as a function of time, where the time parameter varies between 0 and 1 , corresponding to exhalation and inhalation respectively. The figure shows a clear minimum for both thin-plate spline deformation and linear interpolation, with minima of $2.2 \mathrm{~mm}$ and $2.6 \mathrm{~mm}$ of maximum surface distance. The demon's algorithm gave similar results for maximum surface distances. Computing times were in the range of 50-70 minutes for all three algorithms. 80 control points (manually selected) were used for the control point oriented algorithms in each case. 


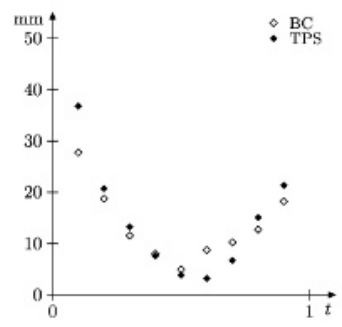

Fig. 4. Maximum surface distance between synthetic and actual image.

\subsection{D Registration}

The fiducial-less tracking technique in section 4 requires a rigid registration of two x-ray images with a series of CT scans. The x-ray-CT registration alone is a six-dimensional registration (three rotational and three translational parameters). To emphasize the fact that a series of CT scans must be registered to a live x-ray image pair with respiration as an additional parameter, we will call this type of registration 7D registration. The experiments consisted of a series of $6 \mathrm{D}$ registrations between x-ray and $\mathrm{CT}$ images. We register two images of sizes $768 / 2 \times 576 / 2$ pixels against a CT image of $512 \times 512$ with 50 slices. (Thus original image size is $768 \times 576$ but only every second pixel was used in both directions for the registration). The original resolution is 3.4 pixels per millimeter, thus 1.7 pixels per millimeter in the images used for registration. Here CT resolution is 1.5 pixel per $\mathrm{mm}$. This low resolution was used, since a large field of view was needed to allow for including a calibration plate. The volume registered was down-sampled to 128 by 128 by 128 voxels and describes a cube of size $128 \times 128 \times 128 \mathrm{~mm}$. The computing times for registration were 5 to 10 seconds with an average number of 150 to 300 function calls to a Hooke-Jeeves patterns search minimizer [3]. Hardware-accelerated volume rendering via $2 \mathrm{D}$ textures is used. Similarity measurement with the correlation coefficient gave best results over a variety of other similarity measures such as MI, NMI (see [5]). In order to lower the influence of regions outside the volumes to be registered, the following procedure was used: the rendered volume was threshold-segmented, and enlarged by 5 pixels via dilatation. Only the intersection of this $2 \mathrm{D}$ region with the "bulls-eye" stemming from the x-ray images was used for the registration. Computing times refer to a Pentium 3 processor with $1400 \mathrm{Mhz}$, running under Linux with $512 \mathrm{MB}$ main storage and a 3D-card NVidia GeForce2.

\section{Discussion and Conclusions}

Clinical testing of the method in section 3 demonstrates an overall correlation error of below $2 \mathrm{~mm}$ throughout an entire 70 minutes treatment, while the total target motion was over $10 \mathrm{~mm}$. This suggests that our method meets the defi- 
nition of radiosurgcial accuracy and is capable of reducing safety margins by a very substantial amount.

A registration time of 5-10 seconds for a single simulated registration was determined in our experiments. To find the tomographic image best matching the given live shots, this registration must be repeated for a series of up to 8 synthetic or actual image 3D sets showing intermediate respiration states. Hence a total image processing time of under 100 seconds per live image pair is realistic in the given application. Notice that this would in practice allow for real-time tracking if combined with the correlation technique in section 3 Here 100 seconds is strictly intra-operative time. The reason why this is still fast enough to track is that the tracking relies solely on the external signal, which can be read in real-time.

\section{References}

1. F. L. Bookstein: Principal Warps: Thin-Plate Splines and the Decomposition of Deformations. IEEE Transactions on Pattern Analysis and Machine Intelligence, 11(6):565-587, 1989.

2. M. Fornefett, K. Rohr, H. S. Stiehl: Radial Basis Functions with Compact Support for Elastic Registration of Medical Images. In Proc. Internat. Workshop Biomedical Image Registration (WBIR'99), pages 173-185, Aug. 1999.

3. R. A. Hooke, T. A. Jeeves: Direct Search Solution for Numerical and Statistical Problems. Journal of the ACM, 8(2):230-239, 1961.

4. S. M. Morrill, M. Langer, R. G. Lane: Real-Time Couch compensation for intratreatment Organ Motion: Theoretical Advantages. Medical Physics, 23, 1083, 1996.

5. T. Rohlfing, C. R. Maurer, W. G. O'Dell, J. Zhong: Modeling liver motion and deformation during the respiratory cycle using intensity-based free-form registration of gated MR images. Medical Imaging 2001. In Proc. of SPIE Vol. 4319, pages 337-348, 2001.

6. D. B. Russakoff, T. Rohlfing et. al.: Fast calculation of digitally reconstructed radiographs using light fields. Medical Imaging 2003. In Proc. of SPIE Vol. 5032, 2003.

7. A. Schweikard, G. Glosser, M. Bodduluri, M. Murphy, J. R. Adler: Robotic Motion Compensation for Respiratory Movement during Radiosurgery. Computer-Aided Surgery, 5(4):263-277, 2000.

8. M. R. Sontag, Z. W. Lai et. al.: Characterization of Respiratory Motion for Pedriatic Conformal 3D Therapy. Medical Physics, 23, 1082, 1996.

9. T. Tada, K. Minakuchi et. al.: Lung Cancer: Intermittent Irradiation Synchronized with Respiratory Motion - Results of a Pilot Study. Radiology, 207(3): 779-783, 1998.

10. J.-P. Thirion: Non-Rigid Matching Using Demons. In Proceedings of the 1996 Conference on Computer Vision and Pattern Recognition, pages 245-251. IEEE Computer Society, 1996.

11. S. Webb: The Physics of Three-dimensional Radiation Therapy. Institute of Physics Publishing, Bristol and Philadelphia, 1993. 